\title{
Evaluation of Geovisualization Designs for the Cognition of Movement in Space and Time
}

\author{
Crystal Bae* and Somayeh Dodge \\ University of California, Santa Barbara, \{cbae | sdodge\}@ucsb.edu \\ * Corresponding author
}

Keywords: user evaluation, cognitive visualization, spatiotemporal data, dynamic maps, movement visualization

\begin{abstract}
:
Movement presents unique challenges to visualization as it incorporates a change of location at different spatial scales over varying temporal scales (Fairbairn et al., 2001). In addition, there is a great need for more empirical evaluation of how map displays are interpreted for trajectory and movement flow data (Davies et al., 2013; Dodge, 2020), and how static and dynamic map representations differ. In this cartographic evaluation study, we assess how different static and dynamic geovisualization designs support participants' cognitive understanding of movement parameters such as speed and direction. The study uses trajectory data of tiger movement recorded as a sequence of time-ordered GPS locations in Euclidean space as a use case. To this end, using various visualization techniques and display forms, we assessed how well participants $(n=100)$ identified movement parameters and patterns across trajectory segments or multiple trajectories. Participants were randomly assigned to using either static (map image) or dynamic (map animation) visualizations of various trajectories to complete the evaluation tasks. Study stimuli comprised seven visualization designs that systematically varied the visual variables (White, 2017; Roth, 2017) of shape, color, and size; see Figure 1 below.
\end{abstract}

Each participant completed a set of 10 tasks using each visualization. These tasks comprised identification questions (identify stops, changes in speed, changes in direction, and overall movement direction), descriptions of movement patterns for trajectory segments, and comparisons between segments and tracks (speed, path distance, straight-line distance, and spatial range). Participants were instructed to minimize their time to respond to the evaluation task questions. We found no statistically significant differences in participants' time spent on task questions between the static or dynamic study conditions. Therefore, participants' use of animated track visualizations appeared to be just as efficient for interpreting movement data as compared to using an equivalent static image of the same data. Although duration for task completion was significantly different across some of the visualization designs, time differences were likely due to order effects (and subsequent analyses suggest this is the case).

In terms of accuracy in interpreting movement data, participants' patterns of identifying actions such as stopping, acceleration, and deceleration over the visualized tracks only somewhat differed between conditions. Identification of major changes in direction did not differ by condition for any of the visualizations. For identifying overall direction of movement, we found that estimates were best supported in the static condition by the line-based visualization with speed encoded as both color and thickness (Visualization 3) and the point-based visualization with the underlying line (Visualization 7); in the dynamic condition, direction estimates were best supported by the dot-based visualization with speed encoded by color only (Visualization 4).

In the written descriptions of movement patterns along track segments, there was a fairly high level of agreement across participants. Although some responses can be characterized as incorrect, such as reporting the complete opposite travel direction to that of the actual direction of movement, others show interesting differences in cases where movement parameters can be described subjectively based on the visualization. For instance, in one example segment, over half of the participants' written descriptions state that the animal is speeding up while another third of the descriptions state that the animal is moving at a constant speed. In this case, participants' internal thresholds for what constitutes a change in speed differs, but neither set of responses is objectively correct or incorrect.

Preliminary results of this work demonstrate the value of assessing differences in geovisualization design to support better and more efficient understanding of various movement parameters. Due to the essential spatiotemporal aspects of movement that need to be communicated in the geovisualization of movement data, we compared static and dynamic designs which we systematically varied along the visual variables of shape, size, and color. Participants using the dynamic versions of the visualizations had higher average scores for direction and comparison questions, but differences were not significant. Initial results demonstrate variations in cartographic interpretation with regards to visualization design as well as across participants. For planned future analyses, we will focus on participants' accuracy in their identification of maximum speeds along trajectories and self-reports of their visualization design preferences. 


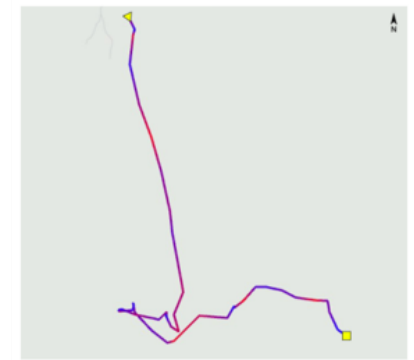

Visualization 1

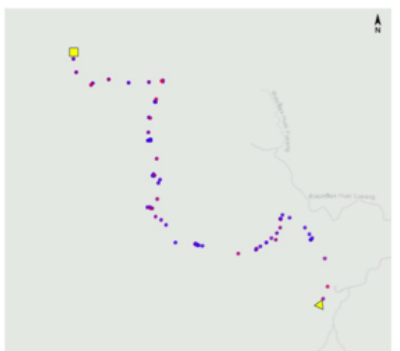

Visualization 4

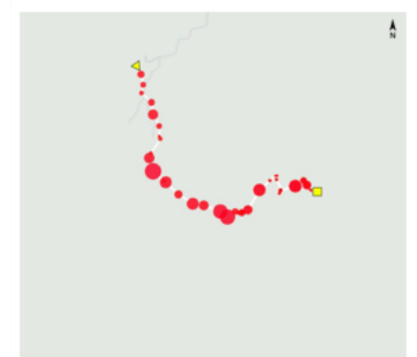

Visualization 7

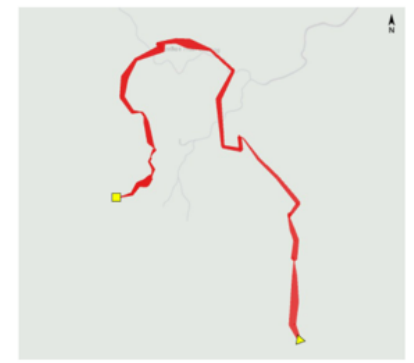

Visualization 2

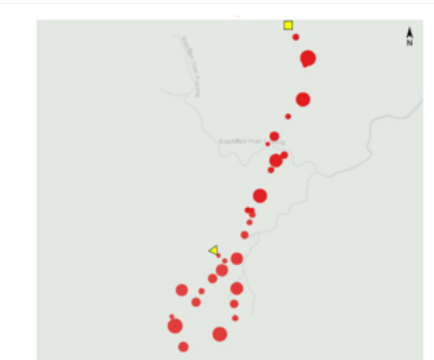

Visualization 5

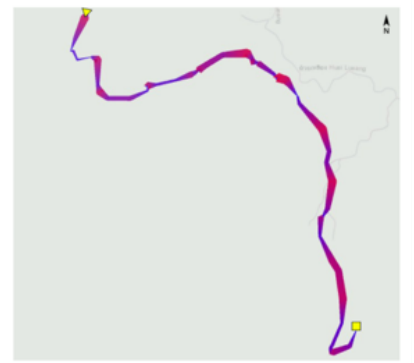

Visualization 3

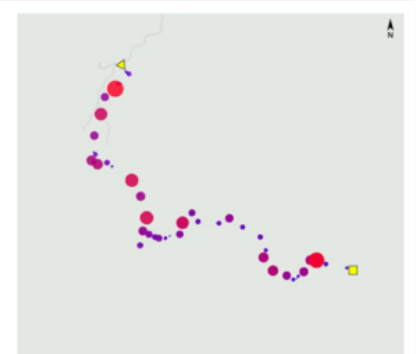

Visualization 6

Figure 1. Visualization designs created using the open source DynamoVis software for movement trajectory visualization (Dodge, Xavier, \& Wong, 2018). 